\title{
Comorbidities in patients with Familial Mediterranean Fever
}

\author{
B Balci-Peynircioğlu' ${ }^{1}$ ZS Arıcl ${ }^{2}, E$ Avcl ${ }^{1}, E D$ Batu², E Arslanoğlu², B Çağlarsu², O Karadağ ${ }^{1}$, U Kalyoncu ${ }^{3}$, Y Bilginer ${ }^{2}$, \\ A Düzova², E Yılmaz', S Özen² \\ From 8th International Congress of Familial Mediterranean Fever and Systemic Autoinflammatory Diseases \\ Dresden, Germany. 30 September - 3 October 2015
}

\section{Introduction}

Familial Mediterranean Fever (FMF) is the most common periodic fever syndrome, characterized by recurrent fever, serositis attacks. There are limited data on comorbidities seen in patients with FMF.

\section{Objective}

Our objective was to evaluate comorbidities among individuals with FMF in a large cohort.

\section{Methods}

We used Hacettepe University- Department of Medical Biology's genetic database records of 5636 FMF patients for MEFV mutation. 1998 patients among this group who are followed by rheumatologists in our hospital, with homozygous and compound heterozygous MEFV mutations were included in the study. We analyzed, Hacettepe University clinical records.

\section{Results}

The mean age was $27,5 \pm 16(1-86)$ years. Our hospital mean follow-up period was 48,5 \pm 48 (1-352) months. 1343 patients $(67,2 \%)$ had no comorbidities. 655 patients (32.8\%) had comorbidities. Comorbidities were as follow: Appendectomy 30 (4,6\%), cholecystectomy 20 (3,1\%), acute rheumatic fever $(2,4 \%)$, ankylosing spondylitis 155 $(23,7 \%)$, juvenile idiopathic arthritis $31(4,6 \%)$, rheumatoid arthritis $10(1,5 \%)$, renal amyloidosis $54(8,2 \%)$, intestinal amyloidosis $4(0,6 \%)$, chronic renal failure 33 (5\%), Behçet's disease $1(0,2 \%)$, Henoch-Schönlein purpura $25(3,8 \%)$, osteoporosis $77(11,8 \%)$, celiac disease $5(0,8 \%)$, inflammatory bowel disease $16(2,4 \%)$, hepatosplenomegaly $33(5 \%)$, hepatosteatosis $20(3,1 \%)$,

${ }^{2}$ Hacettepe University, Pediatric Rheumatology, Ankara, Turkey

Full list of author information is available at the end of the article hypertension $53(8,1 \%)$, polyarteritis nodosa $9(1,4 \%)$, epilepsy $7(1,1 \%)$, multiple sclerosis $5(0,8 \%)$, PFAPA $4(0,6 \%)$, uveitis $1(0,2 \%)$, asthma $6(0,9 \%)$, IgA deficiency $4(0,6 \%)$, thalassemia trait $8(1,2 \%)$, thalassemi major $1(0,2 \%)$, systemic lupus erythematosus $5(0,8 \%)$, sjogren syndrome $2(0,4 \%)$, autoimmune hepatitis $2(0,4 \%)$, autoimmune hemolytic anemia $2(0,4 \%) \ldots$

Homozygous M694V frequency in patients with comorbidities was $42,7 \%$ while it was $34,4 \%$ in those without comorbidities. M694V/M694V mutation in patients with comorbidities was significantly more frequent.

\section{Conclusion}

These comorbidities can be classified in 3 groups: those comorbidities directly related to FMF such as amyloidosis, the second being comorbidities that may be incidental such as IgA deficiency. The last group of comorbidities were those associated with FMF due to increased innate inflammation such as PAN, PFAPA.

It is important to establish the relationship between different diseases' phenotypes and pathologies underlying cellular functions. Thus the results of our study would lead to improved clinical care and epidemiology in FMF.

\section{Authors' details}

${ }^{1}$ Hacettepe University, Medical Biology, Ankara, Turkey. ${ }^{2}$ Hacettepe University, Pediatric Rheumatology, Ankara, Turkey. ${ }^{3}$ Hacettepe University,

Rheumatology, Ankara, Turkey.

Published: 28 September 2015

\section{doi:10.1186/1546-0096-13-S1-P116}

Cite this article as: Balci-Peynircioğlu et al:: Comorbidities in patients with Familial Mediterranean Fever. Pediatric Rheumatology 2015 13(Suppl 1):P116. 